NURSE-SY-2-4

\title{
Selection of immunosuppressive drugs after liver transplantation
}

\author{
DongHwan Jung* \\ University of Ulsan College of Medicine, Seoul, Korea
}

Lecture: Corticosteroids and antimetabolites, with the addition of calcineurin inhibitors (CNIs) a decade later, were the first immunosuppressive agents used among liver transplantation (LT) recipients. They are currently among the most common choices for immunosuppression (IS). Development of the IS after LT allow nowadays to obtain remarkable results. However, lifelong IS is associated with severe adverse events like infectious, cardiovascular, renal, metabolic, and oncologic complications that leads to major morbidity and mortality. Consequently, there is ongoing studies for effective immunosuppressive regimens with acceptable side effects. Newer protocols include combinations of drugs with different modes of action and toxicity profiles. The two prominent approaches are as follows: the upfront strategy in which the immunosuppressive drugs were selected with respect to patient's pretransplant and/or intraoperative risk factors, and the downstream strategy in which a standard therapy is given and changed according to its toxicity and efficacy during the course of therapy. Corticosteroids are employed in immediate and early postoperative period. Although they have a pronounced side effect profile, CNIs are still the backbone of early and late phase immunosuppressive regimens because of their proved efficacy. Antimetabolites are frequent choices for steroid and/or CNI-sparing strategies. Studies also have established a role for mammalian target of rapamycin (mTOR) inhibitors in specific groups of recipients. Biologic agents are a hot topic of interest and made their way into current strategies for induction. IS withdrawal seems feasible in very long-term survivors ( $>10$ years), but is not associated with reversal of IS-related complications. Awaiting novel immunosuppressive drug categories, integration of upfront strategies with the aim to reduce CNI-exposure and a low threshold for adjustment in the posttransplant course are advisable to improve longterm outcomes of LT. 